Pacific Journal of Mathematics

INNER FUNCTIONS INVARIANT CONNECTED COMPONENTS 


\title{
INNER FUNCTIONS INVARIANT CONNECTED COMPONENTS
}

\author{
V. NESTORIDIS
}

The inner functions $d=\exp \{(z+1) /(z-1)\}$ and $z d$ belong to the same connected component in the space of inner functions under uniform topology. Therefore, simplification is not possible in general but it is always possible to simplify by a finite Blaschke product.

o. Introduction. This work deals with the inner functions of one variable. A complex, holomorphic function $f$, bounded on the open unit disk $D$ of the complex plane is called inner if $\left|f\left(e^{i \theta}\right)\right|=1$ a.e.; where $f\left(e^{i \theta}\right)=\lim _{\rho \rightarrow 1} f\left(\rho e^{i \theta}\right)$.

In the set $F$ of the inner functions we consider the topology induced by the Banach space $H^{\infty}$; that is, we consider $F$ with the topology of uniform convergence.

In this work, related to a publication of D. Herrero [2], we are interested in the connected components of the space $F$, mainly with respect to multiplication of inner functions.

Let us denote by $f \sim g$ the fact that the inner functions $f$ and $g$ belong to the same connected component. The questions that motivate this work are the following:

(a) For the identity function $z$, is there an inner function $f$ such that $f \sim z f$ ?

(b) Is simplification permitted? That is, does relation $f \omega \sim g \omega$ imply $f \sim g$ for any three inner functions $f, g, \omega$ ?

The results of this work can be summarized as follow:

(1) "Simplification" by a finite Blaschke product is always possible.

(2) "Simplification" is not possible in general.

(3) If the singular measure $\mu$ associated with a singular function $S$ contains at least one atom, then relation $S \sim z S$ holds.

(4) For any nonconstant inner function $g$, the inner functions $\exp \{(g+1) /(g-1)\}$ and $g \exp \{(g+1) /(g-1)\}$ belong to the same connected component.

(5) For any nonconstant singular function $S$, there exists a nonconstant inner function $g$ such that: $S \sim g S$.

In order to prove that simplification by a finite Blaschke product is possible, we first show that the set $z F=\{z h: h \in F\}=\{x \in F$ : $x(0)=0\}$ is a retract of $F$.

In order to give an example of an inner function $f$ such that $f \sim z f$, we shift the zeros of an infinite Blaschke product in such 
a way that the Blaschke product moves continuously with respect to the uniform topology.

The following problems seem to be open:

(1) Does relation $S \sim z S$ hold for any singular function?

(2) Find all inner functions such that $f \sim z f$.

(3) Characterize the inner functions $\omega$ such that $\omega f \sim \omega g \Rightarrow$ $f \sim g$ for all $f, g \in F$.

(4) Find a necessary and sufficient condition for two inner functions $f$ and $g$ to belong to the same connected component.

1. Preliminaries. A complex, holomorphic function $f$, bounded on the open unit disk $D$ of the complex plane is called inner if its boundary values have almost everywhere absolute volue one; that is, relation $\left|f\left(e^{i \theta}\right)\right|=1$ holds almost everywhere (with $f\left(e^{i \theta}\right)=\lim _{\rho \rightarrow 1} f\left(\rho e^{i \theta}\right)$ ).

It is well-known that a function $f$ is inner if and only if $f$ is of the form:

$$
f(z)=c z^{k} \prod_{i \in I} \frac{\bar{\alpha}_{i}}{\left|\alpha_{i}\right|} \frac{\alpha_{i}-z}{1-\bar{\alpha}_{i} z} \exp \left\{-\int_{0}^{2 \pi} \frac{e^{i \theta}+z}{e^{i \theta}-z} d \mu(\theta)\right\}
$$

where $c$ is a complex constant of modulus one $(|c|=1), k$ is a nonnegative integer, $\mu$ is a positive singular measure on the unit circle and the points $\alpha_{i} \in D$ are such that $\sum_{i \in I} 1-\left|\alpha_{i}\right|<\infty$.

If $\mu=0$, then $f$ is a Blaschke product, finite if the set $I$ is finite or infinite if the set $I$ is infinite (countable).

In the case $I=\varnothing$ and $k=0$, the function $f$ is called singular.

The topology of the uniform convergence on the set $F$ of the inner functions is induced by the following metric:

$$
d(f, g)=\|f-g\|_{\infty}=\sup _{D}|f(z)-g(z)|=\sup _{\theta \in R} \operatorname{ess}\left|f\left(e^{i \theta}\right)-g\left(e^{i \theta}\right)\right| \cdot
$$

Let us denote by $f \sim g$ the fact that the inner functions $f$ and $g$ belong to the same connected component in the space $F$.

In what follows we make use of the well-known facts below:

(1) For any three inner functions $f, g$ and $\omega$ the relation $f \sim g$ implies $\omega f \sim \omega g$. This is due to the continuity of the multiplication of inner functions.

(2) For any inner function $f$ and any complex number $\alpha$, with $|\alpha|<1$, we have the relation:

$$
f \sim f_{\alpha}=\frac{f-\alpha}{1-\bar{\alpha} f}
$$

for the mapping $D \ni \alpha \rightarrow f_{\alpha} \in F$ is continuous.

(3) For every nonnegative integer $n$, the set of all finite Blaschke products with exactly $n$ zeros forms a connected component 
and an open and closed subset of $F$. In particular the set of the constant inner functions is connected and open and closed in $F$.

This fact is an easy application of Rouche's theorem.

2. Simplification by $z$. Let us begin with the question, does the relation $\omega f \sim \omega g$ implies $f \sim g$. This is the problem of "Simplification". In the case of a finite Blaschke product $\omega$, the answer to this question is affirmative.

Proposition 1. Let $\omega$ be a finite Blaschke product. Then for any two inner functions $f$ and $g$, the relation $\omega f \sim \omega g$ implies $f \sim g$.

Proof. The general case easily follows from the case $\omega=z$, to which we will limit ourselves from now on.

Let us consider the set:

$$
z F=\{z h: h \in F\}=\{x \in F: x(0)=0\} .
$$

The maps $z^{*}: z F \rightarrow F$ and $\Phi: F \rightarrow z F$, where $z^{*}(x)=x / z, \Phi(f)=$ $(f-f(0)) /(1-\overline{f(0)} f)$ for $f \in F$ nonconstant and $\Phi(f)=z$ for $f \in F$ constant, are both continuous. (The set of the constant inner functions is, both, open and closed!).

Therefore the mapping $z^{*} \circ \Phi: F \rightarrow F$ is continuous and the relation $z f \sim z g$ implies: $f=z^{*} \circ \Phi(z f) \sim z^{*} \circ \Phi(z g)=g$, as $\Phi(x)=x$ for any $x \in z F$; that is, $\Phi$ is a retraction map and $z F$ is a retract of $F$. The proof is complete now.

3. The main result. The following theorem implies in particular that we cannot "simplify" by any inner function.

THEOREM 1. For any nonconstant inner function $g$, the inner functions $\exp \{(g+1) /(g-1)\}$ and $g \exp \{(g+1) /(g-1)\}$ belong to the same connected component.

This theorem applied for the identity function $g=z(z(a)=a$ for all $a \in D$ ) implies the following:

Proposition 2. The inner functions $d=\exp \{(z+1) /(z-1)\}$ and $z d$ belong to the same connected component (that is: $d \sim z d$ ).

Proposition 2 is equivalent to Theorem 1; for Proposition 2 implies also Theorem 1 . The point is that the range of the continuous map $T_{g}: F \rightarrow H^{\infty}, T_{g}(f)=f \circ g$ is contained in $F$; that is, the 
composition of two inner functions is an inner function ([6] or [8]). Therefore relation $d \sim z d$ implies:

$$
\exp \frac{g+1}{g-1}=T_{g}(d) \sim T_{g}(z d)=g \exp \frac{g+1}{g-1} .
$$

Hence, it remains to prove Proposition 2, which will be a consequence of the following lemma, which is of a concrete geometric nature on the half-plane:

LEMMA 1. Let

$$
K_{1}=\prod_{n=1}^{\infty} \frac{\bar{\alpha}_{n}}{\left|\alpha_{n}\right|} \frac{\alpha_{n}-z}{1-\bar{\alpha}_{n} z} \text { and } K_{2}=\prod_{n=1}^{\infty} \frac{\left|\bar{\beta}_{n}\right|}{\bar{\beta}_{n}} \frac{\beta_{n}-z}{1-\bar{\beta}_{n} z} .
$$

Be two infinite Blaschke products such that $K_{1}(0)>0$ and $K_{2}(0)>0$. If we denote $\varphi(z)=(1+z) /(1-z)$ then we have the following inequality:

$$
\begin{aligned}
\| K_{1}- & K_{2} \|_{\infty} \leqq \sum_{n=1}^{\infty}\left|\alpha \operatorname{rg} \frac{\alpha_{n}}{\beta_{n}}\right|+2 \sum_{n=1}^{\infty}\left|\alpha \operatorname{rg} \frac{1-\alpha_{n}}{1-\beta_{n}}\right| \\
& +2 \sup _{y \in R} \operatorname{ess} \sum_{n=1}^{\infty}\left|\alpha \operatorname{rg} \frac{\varphi\left(\alpha_{n}\right)-i y}{\varphi\left(\beta_{n}\right)-i y}\right| .
\end{aligned}
$$

Proof of Lemma 1. The pointwise convergence $f_{n} \rightarrow f$ implies trivially the inequality:

$$
\|f\|_{\infty} \leqq \liminf _{n}\left\|f_{n}\right\|_{\infty}
$$

We have therefore:

$$
\begin{aligned}
\| K_{1}- & K_{2} \|_{\infty} \leqq \\
= & \lim \operatorname{linf}_{N} \inf _{N}\left\|\prod_{n=1}^{N} \frac{\bar{\alpha}_{n}}{\left|\alpha_{n}\right|} \frac{1-\alpha_{n}}{\left|\alpha_{n}\right|} \frac{\alpha_{n}-z}{1-\bar{\alpha}_{n} z}-\prod_{n=1}^{N} \frac{\bar{\beta}_{n}}{\left|\beta_{n}\right|} \frac{\beta_{n}-z}{1-\bar{\alpha}_{n} z}\right\|_{\infty} \\
& -\prod_{n=1}^{N} \frac{\bar{\beta}_{n}}{\left|\beta_{n}\right|} \frac{\left.1-\beta_{n}\right)+\varphi(z)}{1-\bar{\beta}_{n}} \frac{\varphi\left(\beta_{n}\right)-\varphi(z)}{\bar{\varphi}\left(\beta_{n}\right)+\varphi(z)} \|_{\infty} \cdot
\end{aligned}
$$

We notice that $|\alpha|=|\beta|=\left|\alpha^{\prime}\right|=\left|\beta^{\prime}\right|=1 \Rightarrow\left|\alpha \beta-\alpha^{\prime} \beta^{\prime}\right| \leqq\left|\alpha-\alpha^{\prime}\right|+$ $\left|\beta-\beta^{\prime}\right|$. Consequently, for almost every $z$, with $|z|=1$, we have:

$$
\begin{aligned}
& \left|\prod_{n=1}^{N} \frac{\bar{\alpha}_{n}}{\left|\alpha_{n}\right|} \frac{1-\alpha_{n}}{1-\bar{\alpha}_{n}} \frac{\varphi\left(\alpha_{n}\right)-\varphi(z)}{\bar{\varphi}\left(\alpha_{n}\right)+\varphi(z)}-\prod_{n=1}^{N} \frac{\bar{\beta}_{n}}{\left|\beta_{n}\right|} \frac{1-\beta_{n}}{1-\bar{\beta}_{n}} \frac{\varphi\left(\beta_{n}\right)-\varphi(z)}{\bar{\varphi}\left(\beta_{n}\right)+\varphi(z)}\right| \\
& \leqq \\
& \quad \sum_{n=1}^{N}\left|\frac{\bar{\alpha}_{n}}{\left|\alpha_{n}\right|}-\frac{\bar{\beta}_{n}}{\left|\beta_{n}\right|}\right|+\sum_{n=1}^{N}\left|\frac{1-\alpha_{n}}{1-\bar{\alpha}_{n}}-\frac{1-\beta_{n}}{1-\bar{\beta}_{n}}\right| \\
& \quad+\sum_{n=1}^{N}\left|\frac{\varphi\left(\alpha_{n}\right)-\varphi(z)}{\bar{\varphi}\left(\alpha_{n}\right)+\bar{\varphi}(z)}-\frac{\varphi\left(\beta_{n}\right)-\varphi(z)}{\varphi\left(\beta_{n}\right)+\varphi(z)}\right|
\end{aligned}
$$




$$
\begin{aligned}
& \leqq \sum_{n=1}^{N}\left|\alpha \operatorname{rg} \frac{\alpha_{n}}{\beta_{n}}\right|+2 \sum_{n=1}^{N}\left|\alpha \operatorname{rg} \frac{1-\alpha_{n}}{1-\beta_{n}}\right|+2 \sum_{n=1}^{N}\left|\alpha \operatorname{rg} \frac{\varphi\left(\alpha_{n}\right)-\varphi(z)}{\varphi\left(\beta_{n}\right)-\varphi(z)}\right| \\
& \leqq \sum_{n=1}^{\infty}\left|\alpha \operatorname{rg} \frac{\alpha_{n}}{\beta_{n}}\right|+2 \sum_{n=1}^{\infty}\left|\alpha \operatorname{rg} \frac{1-\alpha_{n}}{1-\beta_{n}}\right|+2 \sup _{y \in R} \operatorname{ess} \sum_{n=1}^{\infty}\left|\arg \frac{\varphi\left(\alpha_{n}\right)-i y}{\varphi\left(\beta_{n}\right)-i y}\right| .
\end{aligned}
$$

The required inequality is now implied.

Proof of Proposition 2. Let $\alpha_{n}(t)$ be the unique point of $D$ such that $\varphi\left(\alpha_{n}(t)\right)=1+i(n+t) \pi$, where $t \in[0,1], n \in N^{*}=\{1,2, \cdots\}$ and $\varphi(z)=(1+z) /(1-z)$.

One, then, verifies easily that:

$$
d_{1 / e}=\frac{d-\frac{1}{e}}{1-\frac{1}{e} d}=f \cdot \prod_{n=4}^{\infty} \frac{\overline{\alpha_{n}(0)}}{\left|\alpha_{n}(0)\right|} \frac{\alpha_{n}(0)-z}{1-\overline{\alpha_{n}(0) z}} \text {, with } f \in F .
$$

It is enough to prove that

$$
B_{1}=\prod_{n=4}^{\infty} \frac{\overline{\alpha_{n}(0)}}{\left|\alpha_{n}(0)\right|} \frac{\alpha_{n}(0)-z}{1-\overline{\alpha_{n}(0) z}} \sim B_{0}=\prod_{n=3}^{\infty} \frac{\overline{\alpha_{n}(0)}}{\left|\alpha_{n}(0)\right|} \frac{\alpha_{n}(0)-z}{1-\overline{\alpha_{n}(0) z}} ;
$$

for, then we have

$$
d \sim d_{1 / e}=f B_{1} \sim f B_{0}=f B_{1} \frac{\overline{\alpha_{3}(0)}}{\left|\alpha_{3}(0)\right|} \frac{\alpha_{3}(0)-1}{1-\overline{\alpha_{3}(0)} z} \sim f B_{1} z=d_{1 / e} \cdot z \sim d z,
$$

and we obtain the result.

In order to prove $B_{1} \sim B_{0}$, it is sufficient to prove the continuity of the following map:

$$
[0,1] \ni t \stackrel{B}{\longmapsto} B_{t}=\prod_{n=3}^{\infty} \frac{\overline{\alpha_{n}(t)}}{\left|\alpha_{n}(t)\right|} \frac{\alpha_{n}(t)-z}{1-\overline{\alpha_{n}(t) z}} \in F ;
$$

that is, $\lim _{t \rightarrow t_{0}}\left\|B_{t}-B_{t_{0}}\right\|_{\infty}=0$ for all $t_{0} \in[0,1]$. Using Lemma 1 we essentially have to prove the following fact:

$$
\lim _{t \rightarrow t_{0}} \sup _{y \in \boldsymbol{R}} \sum_{n=3}^{\infty}\left|\arg \frac{\varphi\left(\alpha_{n}(t)\right)-i y}{\varphi\left(\alpha_{n}\left(t_{0}\right)\right)-i y}\right|=0 \text {. }
$$

This relation follows immediately from the observation that:

$$
\begin{aligned}
& \sum_{n=3}^{\infty}\left|\arg \frac{\varphi\left(\alpha_{n}(t)\right)-i y}{\varphi\left(\alpha_{n}\left(t_{0}\right)\right)-i y}\right| \\
& \quad \leqq 2 \sum_{n=3}^{\infty}\left|\arg \frac{1+2 i \pi\left(n+t_{0}+\left|t-t_{0}\right|\right)-2 i \pi\left(t_{0}+3\right)}{1+2 i \pi\left(n+t_{0}-\left|t-t_{0}\right|\right)-2 i \pi\left(t_{0}+3\right)}\right| \underset{t \rightarrow t_{0}}{\longmapsto} 0 .
\end{aligned}
$$

4. Consequences. Theorem 1 yields trivially the following: 
COROLlaRY 1. For any inner function $g$, there exists an inner function $f$ such that $f \sim g f$.

Proposition 2 implies the following more general result:

CoRollary 2. Let $f$ be an inner function whose singular measure $\mu$ contains at least one atom. Then $f \sim z f$.

Proof of Corollary 2. We have $f=f_{1} \exp K(z+\alpha) /(z-\alpha)$, with $f_{1} \in F,|\alpha|=1$ and $K>0$. Thus, it is enough to establish the relation $\exp K(z+\alpha) /(z-\alpha) \sim z \exp K(z+\alpha) /(z-\alpha)$. By a rotation this becomes:

$$
\exp K \frac{z+1}{z-1} \sim z \exp K \frac{z+1}{z-1}
$$

If $K \geqq 1$, using the known relation $d \sim z d$ (Proposition 2) we have

$$
\begin{aligned}
\exp K \frac{z+1}{z-1} & =d \cdot \exp (K-1) \frac{z+1}{z-1} \sim z d \exp (K-1) \frac{z+1}{z-1} \\
& =z \exp \frac{z+1}{z-1} K .
\end{aligned}
$$

If $0<K<1$, let us consider the transformation ${ }^{1}$ :

$$
-w(z)=\frac{\frac{1-K}{1+K}-z}{1-\frac{1-K}{1+K} z} .
$$

Evidently $w \in F$ and $w \sim z$. From the known relation $d \sim z d$ we obtain:

$$
\exp K \frac{z+1}{z-1}=d \circ w \sim(z d) \circ w=w \cdot(d \circ w) \sim z \cdot(d \circ w)=z \exp K \frac{z+1}{z-1} .
$$

REMARK. Corollary 2 implies that if the singular measure $\mu$ associated with a singular function $S$ contains some atoms, then the relation $S \sim z S$ holds. If the measure $\mu \neq 0$ does not contain any atoms, then we do not know if the relation $S \sim z S$ is true. It seems that this problem (probably not difficult) is still open and we offer the following conjecture:

"Every nonconstant singular inner function $S$ belongs to the same connected component as $z S "$.

\footnotetext{
${ }^{1}$ This trick is found in [2].
} 
In this direction we have the following proposition, which follows from Theorem 1 combined with a remark suggested to the author by K. Stephenson.

Proposition 3. For any nonconstant singular inner function $S$, there exists a nonconstant inner function $g$ such that $S \sim g S$.

Proof. The point is that any singular inner function $S$ is of the form $S=\exp (g+1) /(g-1)$, with $g \in F$. Theorem 1 gives, then, the result.

In an obvious manner Proposition 3 implies the following:

COROLlaRY 3. (i ) For every nonconstant singular inner function $S$, there exist inner functions $f$ and $g$ such that $f S \sim g S$ but $f \nsim g$.

(ii) Let $\omega$ be an inner function such that the relation $f_{1} \omega \sim$ $f_{2} \omega$ implies $f_{1} \sim f_{2}$ for every couple $\left(f_{1}, f_{2}\right)$ of inner functions $f_{1}$ and $f_{2}$. Then the connected component of $\omega$ contains only Blaschke products. In particular $\omega$ is a Blaschke product.

(iii) If the connected component of an inner function $f$ does not contain any proper multiple of $f$, then this component contains only Blaschke products. In particular $f$ is a Blaschke product.

The existence of infinite Blaschke products satisfying the hypothesis of Corollary 3 (iii) follows from the proof of a theorem of D. Herrero ([3], Theorem 1.1). Later, the present author proved in [6] that if the zeros $\alpha_{n}, n=1,2, \cdots$ of a Blaschke product $B$ satisfy the condition

$$
\lim _{n} \prod_{m \neq n}\left|\frac{\alpha_{n}-\alpha_{m}}{1-\bar{\alpha}_{n} \alpha_{m}}\right|=1
$$

then, the connected component for $B$ does not contain any proper multiple of $B$.

ACKNowleDgment. I wish to express my gratitude to A. Bernard for the direction and help he gave me.

\section{REFERENCES}

1. H. Helson, Lectures on the invariant spaces.

2. D. Herrero, Inner functions under uniform topology, Pacific J. Math., 51 (1974), 167-175.

3. - Inner functions under uniform topology II, Revista de la Unión Mathematica Argentina, Volumen 28, 1976. 
4. K. Hoffman, Banach spaces of analytic functions.

5. D. Marshall, Blaschke product generate $H^{\infty}$, Bull. Amer. Math. Soc., 82 (1976).

6. V. Nestoridis, Fonctions intérieures: composantes connexes et multiplication par un produit de Blaschke, Thèse de troisième cycle, Grenoble, (1977).

7. W. Rudin, Real and complex analysis.

8. K. Stephenson, Isometries of the Nevanlina class, Indiana Univ. Math. J., 26 (1977), 307-324.

9. - Omitted values of singular inner functions, to appear, Michigan Math. J.

Received April 7, 1978 and in revised form June 8, 1978.

KALAVRYTON 37

DASSOS HAIDARIOU

Athens, Greece 


\title{
PACIFIC JOURNAL OF MATHEMATICS
}

\section{EDITORS}

DONALD BABBITT (Managing Editor)

University of California

Los Angeles, CA 90024

\section{HUGO RossI}

University of Utah

Salt Lake City, UT 84112

C. C. MOORE and ANDREW OGG

University of California

Berkeley, CA 94720

\section{J. DUGUNDJI}

Department of Mathematics University of Southern California Los Angeles, CA 90007

R. FINN and J. Milgram

Stanford University Stanford, CA 94305

\section{ASSOCIATE EDITORS}
E. F. BECKENBACH
B. H. NeUmanN
F. WOLF
K. YosHIDA

\section{SUPPORTING INSTITUTIONS}

\author{
UNIVERSITY OF BRITISH COLUMBIA \\ CALIFORNIA INSTITUTE OF TECHNOLOGY \\ UNIVERSITY OF CALIFORNIA \\ MONTANA STATE UNIVERSITY \\ UNIVERSITY OF NEVADA, RENO \\ NEW MEXICO STATE UNIVERSITY \\ OREGON STATE UNIVERSITY \\ UNIVERSITY OF OREGON
}

\author{
UNIVERSITY OF SOUTHERN CALIFORNIA \\ STANFORD UNIVERSITY \\ UNIVERSITY OF HAWAII \\ UNIVERSITY OF TOKYO \\ UNIVERSITY OF UTAH \\ WASHINGTON STATE UNIVERSITY \\ UNIVERSITY OF WASHINGTON
}

The Supporting Institutions listed above contribute to the cost of publication of this Journal, but they are not owners or publishers and have no responsibility for its content or policies.

Mathematical papers intended for publication in the Pacific Journal of Mathematics should be in typed form or offset-reproduced, (not dittoed), double spaced with large margins. Please do not use built up fractions in the text of the manuscript. However, you may use them in the displayed equations. Underline Greek letters in red, German in green, and script in blue. The first paragraph or two must be capable of being used separately as a synopsis of the entire paper. Please propose a heading for the odd numbered pages of less than 35 characters. Manuscripts, in triplicate, may be sent to any one of the editors. Please classify according to the scheme of Math. Reviews, Index to Vol. 39. Supply name and address of author to whom proofs should be sent. All other communications should be addressed to the managing editor, or Elaine Barth, University of California, Los Angeles, California, 90024.

50 reprints to each author are provided free for each article, only if page charges have been substantially paid. Additional copies may be obtained at cost in multiples of 50 .

The Pacific Journal of Mathematics is issued monthly as of January 1966. Regular subscription rate: $\$ 84.00$ a year (6 Vols., 12 issues). Special rate: $\$ 42.00$ a year to individual members of supporting institutions.

Subscriptions, orders for numbers issued in the last three calendar years, and changes of address should be sent to Pacific Journal of Mathematics, P.O. Box 969, Carmel Valley, CA 93924, U.S.A. Older back numbers obtainable from Kraus Periodicals Co., Route 100, Millwood, NY 10546.

PUBLISHED BY PACIFIC JOURNAL OF MATHEMATICS, A NON-PROFIT CORPORATION

Printed at Kokusai Bunken Insatsusha (International Academic Printing Co., Ltd.). 8-8, 3-chome, Takadanobaba, Shinjuku-ku, Tokyo 160, Japan.

Copyright (C) 1979 by Pacific Journal of Mathematics Manufactured and first issued in Japan 


\section{Pacific Journal of Mathematics}

\section{Vol. 83, No. $2 \quad$ April, 1979}

Patrick Robert Ahern, On a theorem of Hayman concerning the derivative of a

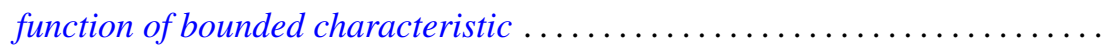

Walter Allegretto, Finiteness of lower spectra of a class of higher order elliptic

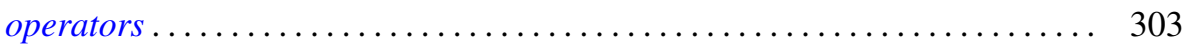

Leonard Asimow, Superharmonic interpolation in subspaces of $C_{c}(X) \ldots \ldots 11$

Steven F. Bellenot, An anti-open mapping theorem for Fréchet spaces . . . . . . . 325

B. J. Day, Locale geometry. . . . . . . . . . . . . . . . . . . . . . . . . . 333

John Erik Fornaess and Steven Krantz, Continuously varying peaking

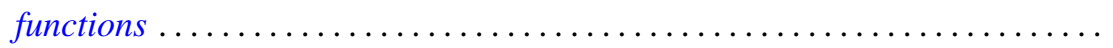

Joseph Leonide Gerver, Long walks in the plane with few collinear points ......

Joseph Leonide Gerver and Lawrence Thom Ramsey, On certain sequences of

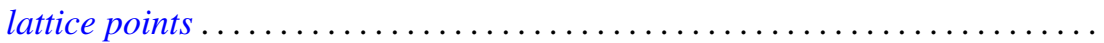

John R. Graef, Yuichi Kitamura, Takaŝi Kusano, Hiroshi Onose and Paul Winton

Spikes, On the nonoscillation of perturbed functional-differential

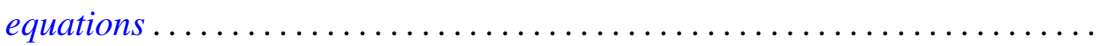

James A. Huckaba and James M. Keller, Annihilation of ideals in commutative

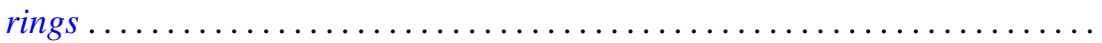

Anzelm Iwanik, Norm attaining operators on Lebesgue spaces . . . . . . . . . . .

Surjit Singh Khurana, Pointwise compactness and measurability . . . .......... 387

Charles Philip Lanski, Commutation with skew elements in rings with

involution.

Hugh Bardeen Maynard, A Radon-Nikodým theorem for finitely additive bounded

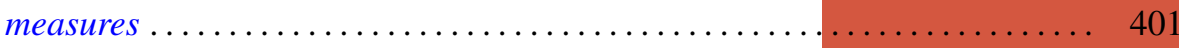

Kevin Mor McCrimmon, Peirce ideals in Jordan triple systems ..

Sam Bernard Nadler, Jr., Joseph E. Quinn and N. Stavrakas, Hyperspaces of compact convex sets.

Ken Nakamula, An explicit formula for the fundamental units of a real pure

sextic number field and its Galois closure ............

Vassili Nestoridis, Inner functions invariant connected components . .

Vladimir I. Oliker, On compact submanifolds with nondegenerate parallel

normal vector fields.

Lex Gerard Oversteegen, Fans and embeddings in the plane.

Shlomo Reisner, On Banach spaces having the property G.L

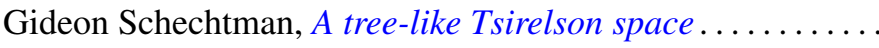

Helga Schirmer, Fix-finite homotopies . . . . . . . . . . . .

Jeffrey D. Vaaler, A geometric inequality with applications to linear forms . .

William Jennings Wickless, $T$ as an $\mathscr{G}$ submodule of $G$.....

Kenneth S. Williams, The class number of $Q(\sqrt{-p})$ modulo 4 , for $p \equiv 3$ (

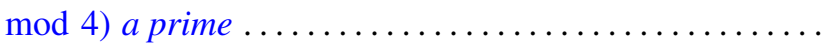

